\title{
IMPEDIMETRIC PLANT BIOSENSOR BASED ON MINIMALLY INVASIVE AND FLEXIBLE MICRONEEDLE ELECTRODES
}

\author{
Abdullah Bukhamsin, Khalil Moussi, Niketan Patel, Alexander Przybysz, Yajun Wang, Simon \\ Krattinger, Jürgen Kosel \\ King Abdullah University of Science and Technology, Thuwal, Saudi Arabia
}

\begin{abstract}
This paper presents an impedimetric biosensor for monitoring of crops using novel microneedle electrodes for minimally invasive, sensitive measurements, compatible with smart agriculture developments. The biosensor was fabricated through polydimethylsiloxane imprinting using a high-resolution 3D printed negative mold. This method allows for fabrication of tailored microneedles with heights up to $500 \mu \mathrm{m}$, required to penetrate through the epidermis of a leaf. The sensor was tested by recording the bioimpedance of Barely leaves and comparing its performance to conventional planar and needle electrodes. Furthermore, we present evidence of a diurnal pattern in plant bioimpedance that was detected by monitoring the leaf bioimpedance under controlled conditions.
\end{abstract}

\section{KEYWORDS}

Precision Farming, Crop Monitoring, Plant Biosensor, Electrical Impedance Spectroscopy.

\section{INTRODUCTION}

Ensuring food security for the steadily growing global population is becoming increasingly difficult. By 2050, the UN projections show that the food production levels need to increase by $70 \%$ from their level in 2007 to meet the demand [1]. Although there is a sufficient global spare capacity for food production, it remains untapped. In part, this is due to the emerging resource constraints, which render the conversion of more land for agriculture unsustainable and economically unfeasible [1]. As such, considerable attention has been dedicated to either increasing or maintaining the yields of the existing fields without increasing their environmental footprint.

One such approach to achieve this daunting goal is precision farming (PF), which seeks to link the spatial and temporal needs of crops to site-specific supplies [2]. This approach has the potential to increase field productivity while mitigating the consumed resources by farming. PF techniques translated to agricultural systems have already resulted in higher yields at a reduced supply of water and fertilizers [2,3]. It is believed that the adoption of PF techniques can be greatly accelerated with the availability of precise and robust sensors that allow for real-time, rapid, and non-destructive monitoring of crops [2].

One such class of sensors are impedimetric sensors. By measuring the bioimpedance of plant tissues, it is possible to detect a variety of biotic and abiotic stress factors. Previously, it was reported that the bioimpedance of plant tissues changes at the onset of fungal infestation [4], temperature stress [5], and bruising [6]. However, impedimetric sensors have yet to see wide-spread adoption in commercial farming. Commonly used needle electrodes are too invasive and impractical for many applications. As for planar electrodes, like skin, the upper layer of plant leaves is coated with a waxy cuticle layer [7], which insulates the underlying tissue and limits the information that can be extracted, when probing the leaves. As such, the sensitivity suffers, and it is difficult to discern if the source of change in bioimpedance is due to a stress factor [4].

To circumvent this barrier, we have developed conformal and lightweight impedimetric biosensor equipped with microneedles (MNs) that can penetrate the cuticle to probe the bioimpedance of leaves with minimal damage and leaf deflection. The biosensor was fabricated using a polydimethylsiloxane (PDMS) imprinting method and a 3D printed negative mold [8]. We demonstrate the versatility of this method in the fabrication of MNs with heights up to $500 \mu \mathrm{m}$ and high fidelity to the desired MNs geometry.

We investigated the performance of the $\mathrm{MN}$ electrodes compared to planar electrodes in monitoring the bioimpedance of Barley leaves in a controlled environment. This comparison showed that the impedance results of the MN-equipped electrodes in the $\alpha$-dispersion region, which are not well-understood, are similar to that of the invasive Pt-needle electrodes. Furthermore, longterm monitoring of the bioimpedance of the Barley leaf using the $\mathrm{MN}$ electrodes under controlled temperature and lighting cycles has revealed evidence of a diurnal pattern in the impedance at low frequencies which may be entrained by light.

\section{SENSOR DESIGN}

The thickness of the cuticle can range from submicrons up to $10 \mu \mathrm{m}$ depending on the hydration state and age of the leaf bud [7]. Under the cuticle, lies the epidermis and the mesophyll, in which all photosynthetic activities take place [7]. To probe the bioimpedance of Barley leaves from these layers, the electrode was fabricated with an array of $100 \mathrm{MNs}$ whose shaft length is $230 \mu \mathrm{m}$ and the diameter is $240 \mu \mathrm{m}$. The spacing between the MNs on the array was $360 \mu \mathrm{m}$ to minimize the "bed-ofneedles" effect.

To ensure optimal contact between the sensor and the leaf while measuring the impedance, the sensor had to be tailored to be sufficiently conformal to the surface. To that end, the PDMS layer used as a platform to support the MNs was designed to be $350 \mu \mathrm{m}$ thick. This allowed the sensor bed to adhere to the leaf whilst being light in weight as to minimally bend the leaf. Furthermore, owing to its hydrophobic nature, the PDMS bed also helps in preventing excessive plant transpiration from the micropores created by the puncturing action of the MNs.

As PDMS has a relatively low elastic modulus, PDMS MNs are prone to bend or buckle under an axial load. A thin metallic coating sputtered on the MNs would not be sufficient to obtain the needed mechanical integrity. This would prevent the MNs from piercing the surface of the 
leaf. To alleviate the issue, a thicker layer of about $5 \mu \mathrm{m}$ of Nickel was electroplated on a Titanium seed layer of $40 \mathrm{~nm}$ covering the MNs. The plated Nickel increases the mechanical integrity of the MNs. However, since Nickel is also a micronutrient for plants, using Nickel based electrodes may illicit a biological response and alter the bioimpedance signal. Thus, we opted to coat the Nickel with a $150 \mathrm{~nm}$ sputtered layer of Gold (Fig 1).

\section{FABRICATION}

The master mold fabrication begins by dicing a singlesided polished p-type 4" Silicon wafer to $2.5 \times 2.5 \mathrm{~cm}^{2}$ substrates. The diced substrates are then sequentially cleaned by acetone, isopropanol, DI water and then dried with Nitrogen. IP-S resin (Nanoscribe, $\mathrm{GmbH}$ ) is then drip casted onto the substrate, which is then loaded into a direct laser lithography system (Photonic Professional GT, Nanoscribe, $\mathrm{GmbH})$. The objective lens (25x, NA 0.8) is then immersed into the resin. The Silicon-resin interface is determined by the mismatch in their refractive indices. The mold is defined by the IR laser's focal point at which twophoton polymerization is triggered. To achieve the desired micro-scale resolution and mechanical integrity of the mold needed to survive the PDMS demolding process, the mesoscale shell mode was selected with the laser power set to $100 \mathrm{~mW}$.

The mold was then developed by submersion into mrDEV 600 (micro resist technology, $\mathrm{GmbH}$ ) for 25 minutes, then into isopropanol for 1 minutes, and dried with a gentle stream of Nitrogen. To smooth out the stitching lines on the mold and to secure it onto the substrate, a $3 \mu \mathrm{m}$ layer of Parylene $\mathrm{C}$ was deposited on the mold (Specialty Coating System, KISCO). A frame of Polymethyl methacrylate is laser cut using a $\mathrm{CO}_{2}$ laser and is secured around the mold using an instant adhesive (LOCTITE 401). This frame is used to define the surface area of the sensor and the thickness of the PDMS bed.

The PDMS (Sylgard 184) to be imprinted is prepared by mixing the PDMS and the curing agent in a 10:1 weight ratio. The mixing generates bubbles that must be outgassed by desiccation for 45 minutes prior to pouring it over the mold. The PDMS is then decanted over the mold and a surgical blade is used to remove the excess. As air bubbles may be trapped at the cavities defining the MN bores, the PDMS poured over the mold must be outgassed again to allow the trapped bubbles to be released and for the PDMS to flow completely into the bottom of the bores. The mold is then inserted into a convection oven set at $90^{\circ} \mathrm{C}$ to cure the PDMS for 30 minutes. A surgical blade is then used to release the PDMS from the mold and the PMMA frame.

As PDMS has poor adhesion to metal, the PDMS MNs is treated with $\mathrm{O}_{2}$ plasma for 30 seconds, prior to the deposition of the Titanium seed layer. This plasma ablation increases the mean surface roughness by $20 \mathrm{~nm}$ [9]. The increased contact area improves the adhesion between PDMS and Titanium. Subsequently, $40 \mathrm{~nm}$ of Titanium is sputtered on the MNs. A layer of $5 \mu \mathrm{m}$ of Nickel is then electroplated on the MNs by submerging the specimens in a solution with soluble Nickel salts and applying a voltage of $1.8 \mathrm{~V}$ between the specimens and the anode for 5 minutes. Finally, a layer of $150 \mathrm{~nm}$ of Gold is sputtered on the specimens and contacts are soldered onto the bed.

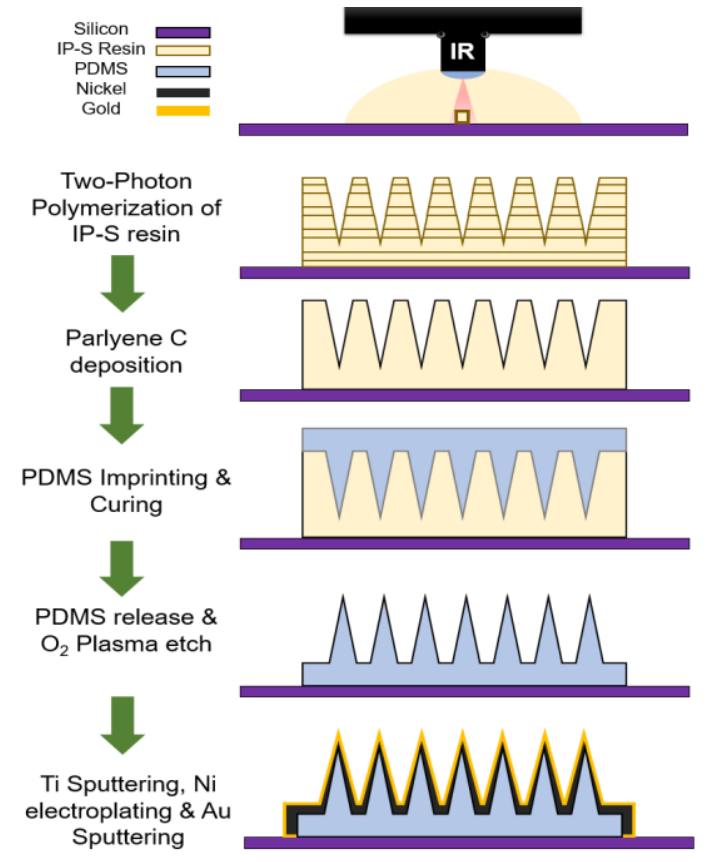

Figure 1: A schematic overview of the fabrication process flow for the impedimetric sensor.

\section{MECHANICAL TESTING}

To assess the maximum axial load that MNs can withstand, we adapt a displacement-force testing setup as used by [10]. A $10 \mathrm{~N}$ load cell of an Instron 5966 mechanical testing system is used to press a MN array against a solid metal surface (Fig 2) to generate a loaddisplacement curve. The displacement rate was set to 20 $\mu \mathrm{m} /$ second and data acquisition continued until all MNs were bent, signifying failure. The MNs were then inspected under the microscope to determine the mode of failure.

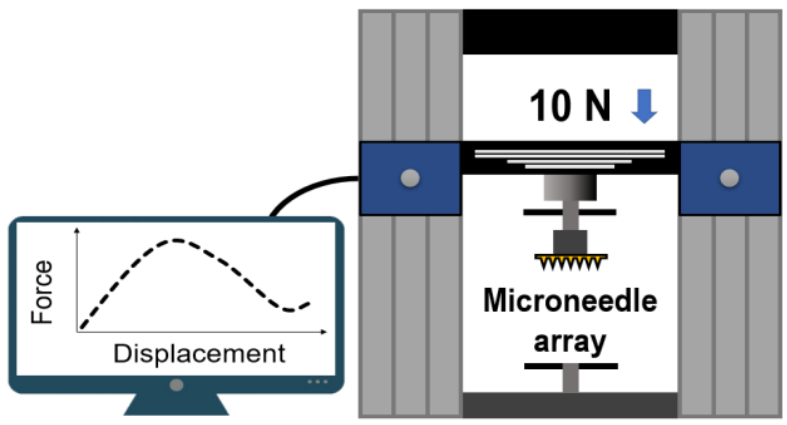

Figure 2: Mechanical test setup.

\section{BARLEY IMPEDANCE MEASURMENTS}

To eliminate the effect of confounding environmental variables on the measured bioimpedance, the Barely specimens were placed in a growth chamber under a controlled temperature and day/night cycle. The plants received 12 hours of light at a temperature of $22^{\circ} \mathrm{C}$ and 12 hours of darkness at a temperature of $16^{\circ} \mathrm{C}$ (Fig 3). During the assessment, the soil was unchanged and the water levels in the tray were maintained at a static level. A pair of sensors were attached to the blade of the leaf and were fixed using 3M surgical tape, which has a porous structure allowing the leaf to be aerated (Fig 3).

The bioelectric impedance of Barley leaves was assessed at frequencies between $1 \mathrm{~Hz}$ to $100 \mathrm{kHz}$ using a portable potentiostat (PalmSens4, PalmSens BV). The AC 
voltage used to probe the bioimpedance was fixed at 35 $\mathrm{mV}$. The inter-electrode distance was varied between $1 \mathrm{~cm}$, $2 \mathrm{~cm}$, and $3 \mathrm{~cm}$ to confirm the reliability of the measured bioimpedance. The same site on each leaf was inspected twice; once using planar Gold electrodes and once using the MNs-electrodes. Insertion site parity between the MNselectrodes and the conventional Platinum needle electrodes cannot be achieved on Barley, as the needles would simply penetrate through the full thickness of the leaf. As such, Platinum needle electrodes were inserted into two adjacent fronds and the bioimpedance was recorded.

To investigate the effect of lighting on the bioimpedance of Barley leaves, the MNs-electrodes were attached with a spacing of $3 \mathrm{~cm}$ (Fig 3). The potentiostat was set to record the bioimpedance at intervals of 15 minutes and the experiment was conducted in the growth chamber for the duration of 7 days.

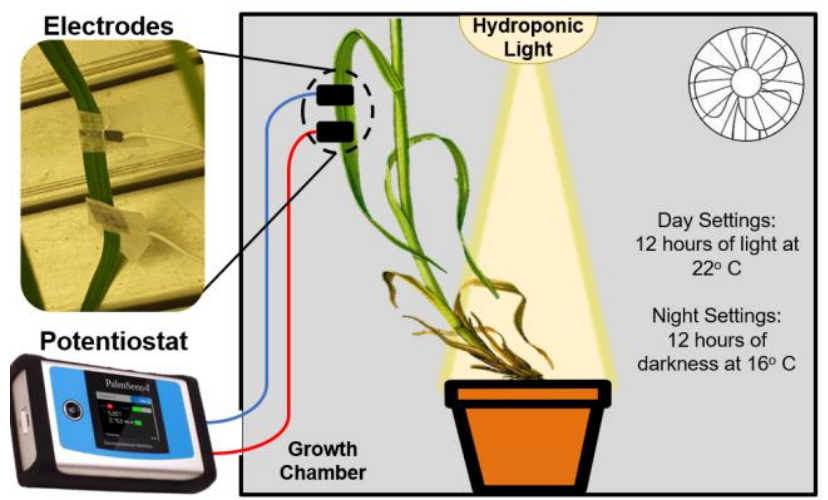

Figure 3: Measurement setup used to acquire the bioimpedance data.

\section{RESULTS}

Using two photon polymerization, we fabricated two molds of MNs arrays with shaft lengths of $500 \mu \mathrm{m}$ and 230 $\mu \mathrm{m}$ with base diameters of $240 \mu \mathrm{m}$. The molds were used to imprint PDMS as outlined in (Fig 1) and the resulting MNs arrays were inspected under a scanning electron microscopy (SEM) (Fig 4). The SEM images show the high fidelity preserved by the imprinting process onto the PDMS as demonstrated by the visibility of the stitching lines on the PDMS.

As shown in (Fig 5), the MNs in the array bend at an applied force of $132 \mathrm{mN} \pm 27 \mathrm{mN}$. The atypical compression curve can be explained by the elastic properties of PDMS. At the point of contact with the metal surface, the MNs begin to bend gradually, thereby deforming the Nickel shell. At an average force of $132 \mathrm{mN}$, the MNs invert onto the PDMS bed and the Nickel shell is cracked. This allows for the metal plate to compress the PDMS MN at a nearly constant force. After the MNs are completely inverted onto the bed, the metal plate begins to compress the PDMS bed itself and the force needed for that compression rises. The cracking of the Nickel shell was confirmed by inspecting the MNs by SEM after failure (inset Fig 5). The failure point may be tuned by depositing a thicker layer of Nickel.

Measuring the bioimpedance of Barley leaves using a planar electrode and MNs-electrodes, yields a stark difference in the reported impedance at frequencies between $1 \mathrm{~Hz}$ to $50 \mathrm{kHz}$ (Fig 6). At higher frequencies, the impedance as measured by the two electrodes converges. This is because at frequencies above the $50 \mathrm{kHz}$ mark, the capacitive layers of the cuticle and plant cellular walls act as shorts to the passage of current. Thus, both the intracellular and extracellular fluids contribute to the measured impedance at that point.
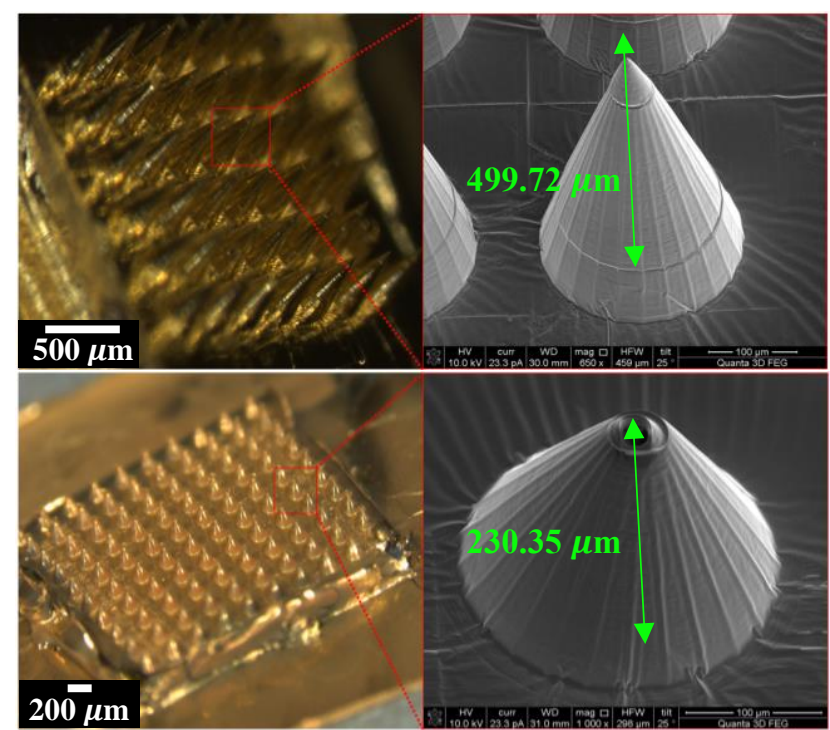

Figure 4: PDMS MN imprint after curing and coating (left) and a representative $M N$ imaged by SEM (right) for the fabricated molds.

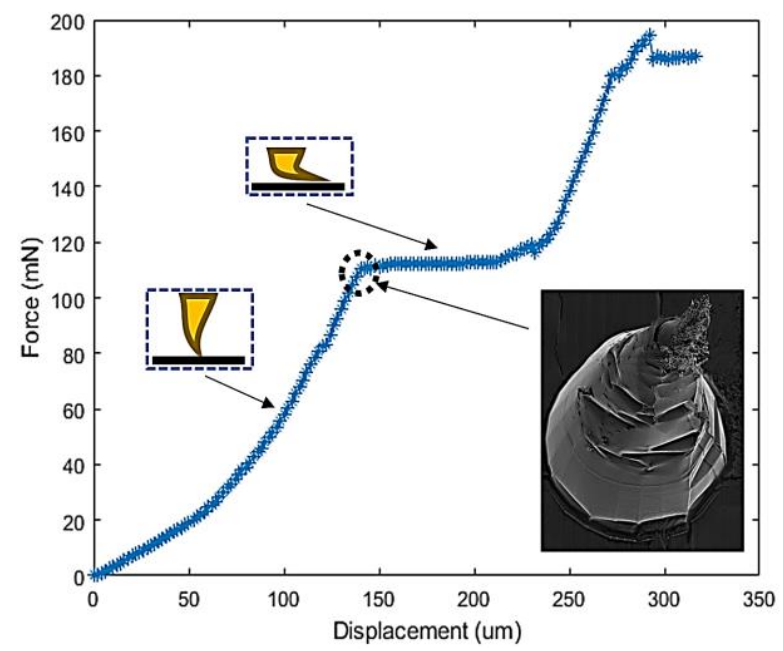

Figure 5: Load-displacement curve generated via crash testing the MN array. Insets contain the geometry of the MNs during compression and show the cracked shell.

Although a direct comparison to the Platinum needle electrodes is not possible due to differences in the insertion sites, the measured bioimpedance using the needle electrodes is lower than both the planar and MNselectrodes in the $1 \mathrm{~Hz}$ to $50 \mathrm{kHz}$ interval. However, the impedance reported by the Platinum needle electrodes at frequencies higher than $50 \mathrm{kHz}$ is higher than the other two configurations due to the longer current path associated with their insertion sites.

Long term monitoring of the bioimpedance of Barley leaves using the MNs-electrodes produced evidence for a diurnal cycling of impedance in the $\alpha$-dispersion region (Fig 7). The growth chamber used for this experiment 
cycles between 12 hours of light and 12 hours of complete darkness, without any graduation of light between the two intervals. The bioimpedance sharply increases at the onset of the lights being turned on and falls sharply when they are turned off.

One possible explanation is the dynamics of water movements in and out of the leaf throughout the day. During the day, the photosynthetic activity is at its peak, and the leaf acts as a sink for water, translating to a constant flux of water in and flow of sugars out through the phloem, which may explain the rise in impedance during the day. Conversely, at night, there is an osmotic balance between the leaf and the stem, opposing any tendency for the water to flow, maintaining a relatively low impedance relative to the day.

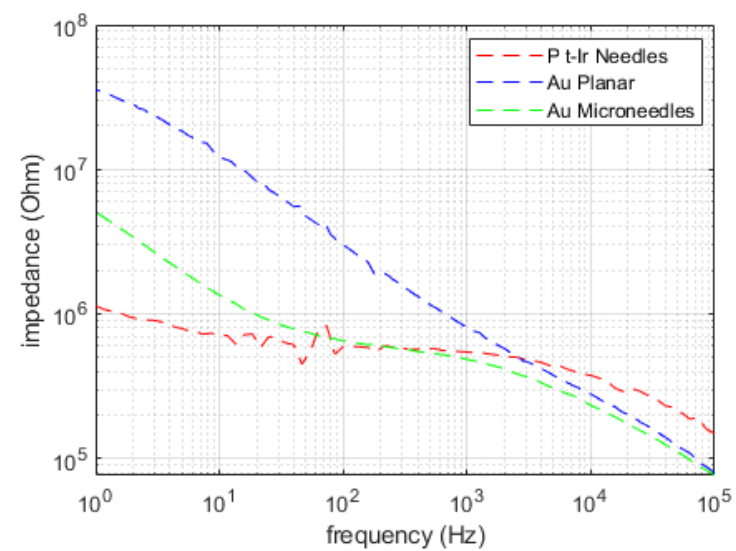

Figure 6: Bode plots of Barely leaves as acquired by Platinum needle (red), MNs (green) and planar (blue) electrodes.

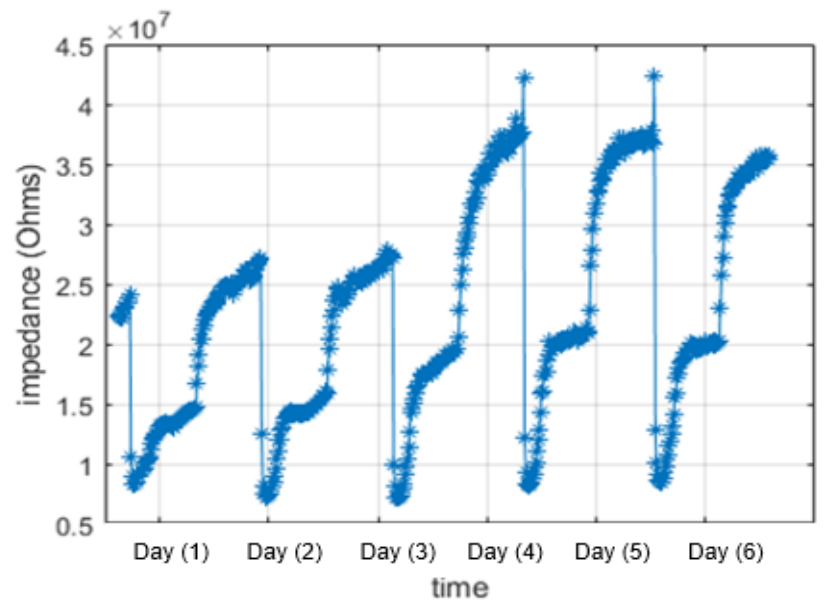

Figure 7: Cyclic Impedance of Barely leaf at $1 \mathrm{~Hz}$. The impedance rises after the day (3), which is possibly due to the resealing of the leaf around the MNs.

\section{CONCLUSION}

In this work, we have developed a fabrication process for flexible, lightweight microneedle plant electrodes to enable minimally invasive yet highly sensitive impedance measurements. The electrodes are made of PDMS as a substrate material with metal coating to increase the mechanical strength. We utilized imprinting in a 3D printed mold, combining the versatility in design of the $3 \mathrm{D}$ printing with fabrication scalability. We demonstrate the potential of these electrodes for impedimetric biosensing by monitoring crops. Furthermore, we presented evidence of a diurnal cycling in the bioimpedance of Barley at low frequencies that appears to be entrained by light. Future work will focus on utilizing this technique to tabulate the response of Barley and other commercially important crops to variety of biotic and abiotic stress and will investigate the possibility of monitoring specific phytohormones by functionalizing the MNs. We aim to provide an analytical platform for the complete assessment of crops health.

\section{ACKNOWLEDGEMENTS}

The authors thank the staff of the Nanofabrication core lab at King Abdullah University of Science \& Technology. This work was funded by King Abdullah University of Science and Technology and the RPW project grant from the Office of Sponsored Research

\section{REFERENCES}

[1] A. Nikos, and J. Bruinsma. "World Agriculture Towards 2030/2050", FAO, 2012.

[2] B. Athanasios, et al. "Precision Agriculture Technologies Positively Contributing to GHG Emissions Mitigation, Farm Productivity and Economics", Sustainability, vol. 9, no. 8, 2017, pp. 1339-1367.

[3] P. Reyns, et al. "A Review of Combine Sensors for Precision Farming", Precision Agriculture, vol. 3, 2002, pp. 169-182.

[4] K. Alfadhl, et al. "Early Detection of Diseases in Plant Tissue Using Spectroscopy - Applications and Limitations.” Appl. Spectroscopy Rev., vol. 53, no. 1, 2017, pp. 36-64.

[5] J. Ildikó, et al. "Electrical Impedance Measurement on Plants: A Review with Some Insights to Other Fields." Theoretical and Experimental Plant Physiology, vol. 31, no. 3, 2019, pp. 359-375.

[6] E. Borges, et al. "Early Detection and Monitoring of Plant Diseases by Bioelectric Impedance Spectroscopy." IEEE 2nd Portuguese Meeting in Bioengineering, 2012.

[7] D. Eva, et al. "The Biophysical Design of Plant Cuticles: An Overview." New Phytologist, vol. 189, no. 4, 2010, pp. 938-949.

[8] K. Moussi, and J. Kosel, "3-D Printed Biocompatible Micro-Bellows Membranes", J. Microelectromech. Syst., vol. 27, no. 3, 2018, pp. 472-478.

[9] B. Yeoul, et al. "Stable Deposition and Patterning of Metal Layers on the PDMS Substrate and Characterization for the Development of the Flexible and Implantable Micro Electrode." Solid State Phenomena Advances in Nanomaterials and Processing, 2007, pp. 165-168.

[10]K. Moussi, et al. "Biocompatible 3D Printed Microneedles for Transdermal, Intradermal, and Percutaneous Applications", Adv. Eng. Mater., 2019.

\section{CONTACT}

*A. Bukhamsin: abdullah.bukhamsin@kaust.edu.sa

*J. Kosel: jurgen.kosel@kaust.edu.sa 